\title{
167. Time Course Analysis of Cation Influxes during the Prolonged Action Potential in Prefused Squid Giant Axon
}

\author{
By Toshifumi Takenaka and Katsuto Yumoto \\ Department of Physiology, Tokyo Medical and Dental University, \\ Yushima, Bunkyo-ku, Tokyo \\ (Comm. by Yas Kuno, M. J. A., Oct. 13, 1969)
}

The production of an action potental in the squid giant axon is associated with enhancement of influxes of univalent and divalent ions. ${ }^{2,3), 7)}$ However, it has been difficult to decide with certainty in what phase of bioelectric activity this enhancement occurs, because the radiotracer technique is limited by poor time resolution. Such difficulties are partially overcome in this experiment by using a prolonged action potential and a moving-autoradiographic method. The axon membrane produces a prolonged action potential measured in seconds under continuous perfusion with $\mathrm{CsF}$ solution internally and $\mathrm{CaCl}_{2}$ solution or combined $\mathrm{CaCl}_{2}$ and $\mathrm{NaCl}$ solution externally. ${ }^{7}$ )

This paper describes the cation influx during the plateau phase, and the time course of radioisotope influx during the prolonged action potential by using moving autoradiography from which it can be seen that the amount of cation influx remains at the same elevated value during the prolonged action potential.

Methods. Squid giant aoxns obtained from Doryteuthis kensaki. available at Wakasa Bay, Kyoto, were used for these experiments. The axon diameters were approximately $500 \mu$. A Lucite perfusion chamber, $30 \mathrm{~mm}$ in width, supported the axon which was exposed to artificial solutions in the central $20 \mathrm{~mm}$ portion of the chamber. The unperfused lateral segments of the axon were separated from the middle position by white petroleum jelly.

A glass cannula was inserted into each end of the axon and internally perfused by a technique used previously.4),5),8) The tip of the smaller inlet cannula measured $180 \mu$ in diameter and was placed concentrically within the lumen of the larger outlet cannula measuring $320 \mu$ in diameter. Internal perfusion was initiated by separating the two cannulae. The length of the internal perfusion zone was about $20 \mathrm{~mm}$. The inlet cannula was connected to a reservoir of perfusion fluid by polyethylene tubing.

The internal perfusing solutions were usually prepared by mixing a glycerol solution at 12 vol. \% with $600 \mathrm{mM}$ CsF and adjusting the 
$\mathrm{pH}$ of the solution to 7.2 with a small amount of Cs-phosphate buffer. The CsF concentration was $25 \mathrm{mM}$ in most cases. In order to remove the axoplasm, the axon was perfused with $25 \mathrm{mM}$ CsF-glycerol solution containing $0.1 \mathrm{mg} / \mathrm{ml}$ Pronase for a period of $2 \mathrm{~min}$ and followed by an enzyme-free solution. $\left.{ }^{7)}, 8\right)$ The flow rate of the internal perfusate was maintained at $12.6 \times 10^{-3} \mathrm{ml} / \mathrm{min}$. The external fluid medium was prepared by mixing $400 \mathrm{mM} \mathrm{CaCl} 2$ with an approximately isosmotic glycerol solution (12 vol.\%). External solutions containing $\mathrm{NaCl}$ and $\mathrm{CaCl}_{2}$ were prepared by mixing $400 \mathrm{mM} \mathrm{CaCl}, 600 \mathrm{mM}$ $\mathrm{NaCl}$, and 12 vol.\% glycerol solution. The $\mathrm{pH}$ of these media were adjusted to 8.0 with a small amount of Tris-HCl buffer.

Two twisted platinum wire electrodes were used as internal electrodes: one for recording and another for supplying the stimulating current. The current electrode was made of $50 \mu$ enamelled wire, with two bare portions measuring $5 \mathrm{~mm}$ in length located near the tip. The recording electrode was twisted around the current electrode, and a bare region of $2 \mathrm{~mm}$ in length was placed midway between the two bare portion of the current electrode. Both electrodes were carefully coated with platinum black by electrolysis of a chloroplatinic acid solution. A saturated $\mathrm{KCl}$-calomel electrode placed in the bathing fluid was used as a reference electrodes.

Cation influxes were measured by adding radioisotopes, $\mathrm{Na}^{22}$ and $\mathrm{Ca}^{45}$, to the external fluid medium. $\mathrm{Na}^{22}$ was obtained from Daiichi Pure Chemical Co. Ltd. in the chloride form. Radioactive calcium, $\mathrm{Ca}^{45}$, was supplied by International Chemical and Nuclear Corp. Samples of the internal perfusion fluid were then collected over each 5 min period from the outlet cannula and were dried in planchets before counting. Radioactivity was determined with a low background counter, Widebeta II Beckman. The background radio activity of this machine was $0.6 \mathrm{cpm}$.

In order to measure the time course of influxes, a paper tape was in contact with the orifice of the outlet cannula and was running with a speed of $1 \mathrm{~cm}$ per sec. driven by a motor as shown in Fig. 1 . The perfusing solution from the outlet cannula was absorbed on one side of the running tape. The tapes were dried in air and placed on photographic emulsion. (Suitable tripping film is available at Fuji Photo. Film Co., Ltd., autoradiographic plates were $5 \times 25 \mathrm{~cm}$.) Exposure time required depends on the isotope and its concentration in the tape. In this experiment, stripping film was exposed up to one month for $\mathrm{Na}^{22}$ and up to two months for $\mathrm{Ca}^{45}$ without excessive background fog developing. All experiments described in this article were carried out at $12 \sim 15^{\circ} \mathrm{C}$.

Results. (1) Influx of ions at rest and during prolonged ac- 


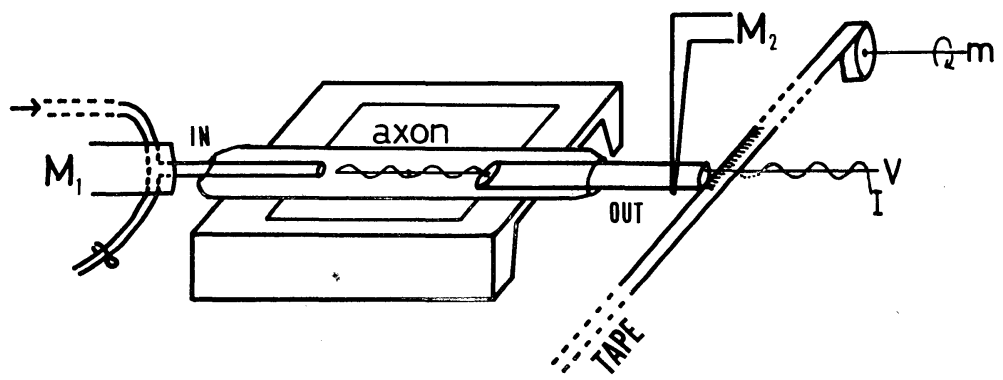

Fig. 1. Schematic diagram illustrating the experimentral arrangement used for intracellular perfusion of Squid giant axon and moving-autoradiography. The inlet cannula (IN) is connected to reservoirs of the perfusion fluid. The outlet cannula (OUT) and the inlet cannula are held with micromanipulators $\left(\mathrm{M}_{1}, \mathrm{M}_{2}\right)$. The internal recording and stimulating electrods $(\mathrm{V}, \mathrm{I})$ are held with separate micromanipulater. The outlet cannula is in contact with the paper tape moved by the rotating motor $(\mathrm{m})$. The outflow of the perfusing solution from the outlet cannula is absorbed at the edge of the paper tape.

tion potentials. The influx of calcium ions into the internal artificial solution was determined. An example of these results is shown in Fig. 2. The internal medium contained $25 \mathrm{mM}$ CsF-glycerol solution. The external medium contained only $100 \mathrm{mM} \mathrm{CaCl}_{2}$-glycerol solution in Fig. 2 left. A tracer amount of ${ }^{45} \mathrm{CaCl}_{2}$ was added to the external medium in which its radioactivity was $8000 \mathrm{cpm}$ for a volume of $10^{-3} \mathrm{ml}$. The internal perfusing solution was collected regularly at the end of each 5 min period. Samples were taken during two resting periods preceding the first stimulation period and two before and following the second stimulation period.

When an axon was subjected to stimulation there was a sharp increase in calcium influx. The first period of stimulation consisted of ten pulses producing prolonged action potentials, each having a duration of about $1.3 \mathrm{sec}$ or a total duration of $13.4 \mathrm{sec}$. In the second stimulus period, fifteen prolonged action potentials occurred and the total duration of these was $8.25 \mathrm{sec}$. The total duration of action potentials during the second stimulation period was about 60 per cent of the total duration of action potentials during the first period, and the calcium influx during the second period was $65 \%$ of the first. It can be seen from these results that the increase of the calcium influx in the axon depends on the plateau phase and not on the number of generated action potentials.

In Fig. 2 right, the axon was perfused internally with $25 \mathrm{mM}$ CsF-glycerol solution and immersed in a solution of $100 \mathrm{mM} \mathrm{CaCl}_{2}$ and $300 \mathrm{mM} \mathrm{NaCl}$. The first stimulation sequence consisted of ten pulses. Each prolonged action potential had on average duration of 


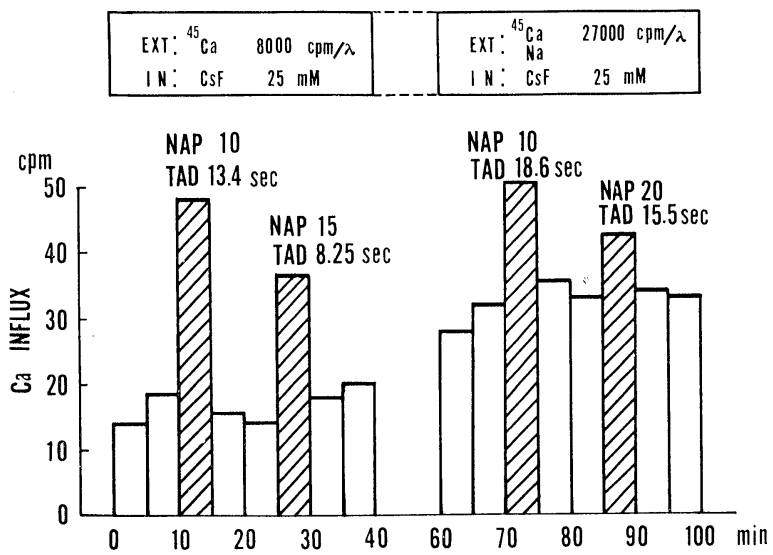

Fig. 2. Calcium influxes into an axon perfused with $25 \mathrm{mMCsF}$ glycerol solution. Ordinate: radioactivity in counts per minute in internal perfusing solution collected at the end of every 5 min period. Abscissa: time after introducing radioisotope. NAP: number of prolonged action potential produced during 5 min. TAD: total action potential duration.

$1.8 \mathrm{sec}$ or total duration of $18.6 \mathrm{sec}$ and an average amplitude of $150 \mathrm{mV}$. The second period of stimulation provided 20 pulses and the average duration of prolonged action potentials was $0.78 \mathrm{sec}$. The total duration of the action potentials was $15.5 \mathrm{sec}$. These results also show that the increase of the calcium influx in the axon depended on the duration of prolonged action potential and not on the number of action potentials generated.

Influxes of radioactive $\mathrm{Na}^{22}$ ion were studied. An example is presented in Fig. 3 in which case the external medium contained an artificial seawater composed of $300 \mathrm{mM} \mathrm{NaCl}$, and $100 \mathrm{mM} \mathrm{CaCl}_{2}$. The $\mathrm{Na}^{22}$ activity was $23000 \mathrm{cpm}$ per $10^{-3} \mathrm{ml}$. The axon was perfused internally with $25 \mathrm{mM} \mathrm{CsF}$-glycerol solution. The prolonged action potential had a duration of about $1 \mathrm{sec}$ and an amplitude of $130 \mathrm{mV}$. At least two resting periods preceded and followed each period of stimulation. The $\mathrm{Na}^{22}$ influx was nearly doubled by stimulation, and the extra influx was again proportional to the duration of the prolonged action potentials similar to what is observed in Fig. 2. In addition, Fig. 2 shows that the change in calcium influx above the resting level on the right is about $1 / 2$ what it is on the left. The cpm of calcium is 3.5 times greater; thus the effectiveness of the calcium influx with $\mathrm{Na}$ present is reduced to $1 / 7$ the value of when $\mathrm{Ca}$ is alone. In Fig. 3, the $\mathrm{Na}$ influx with calcium present is about 3 times what $\mathrm{Ca}$ is alone. The effect on the resting influx is less pronounced. The value of $\mathrm{Ca}$ influx for the resting state with $\mathrm{Ca}$ and Na combined 


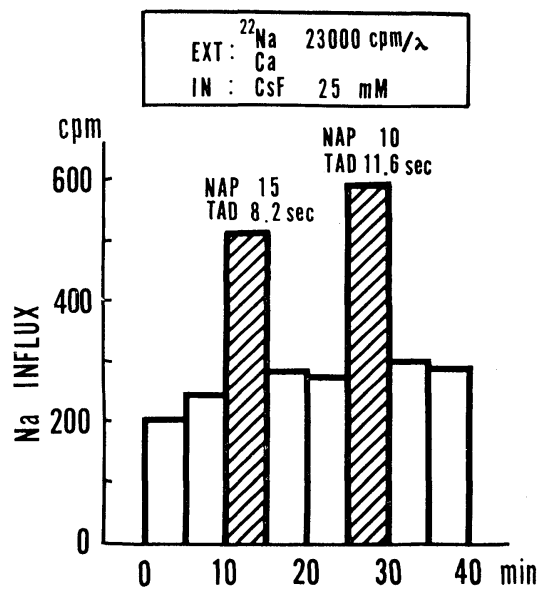

Fig. 3. Sodium influx into an axon perfused with $25 \mathrm{mM}$ CsF-glycerol solution. The external medium is $100 \mathrm{mM}$ $\mathrm{CaCl}_{2}$ and $300 \mathrm{mM} \mathrm{NaCl}$.

is twice the value of $\mathrm{Ca}$ alone.

(2) Autoradiographic approach to time course analysis of influx.

The diagram in Fig. 4 shows an example of the results obtained from an axon perfused with $25 \mathrm{mM}$ CsF-glycerol solution internally. In this example the external solution consisted of $300 \mathrm{mM} \mathrm{NaCl}$ and $100 \mathrm{mM} \mathrm{CaCl}_{2}$. The action potential recorded by this procedure had a duration of $6 \mathrm{sec}$ and is shown in B of Fig. 4. The time course of Na-influx studied by autoradiography is shown in C (see methods). It is seen in this diagram that there is a small influx of sodium before stimulation and that this level of influx was markedly enhanced during the prolonged action potential. There is no appreciable increase in the influx at the onset or the end of the prolonged action

A

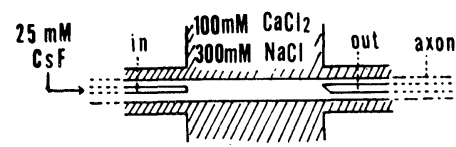

B

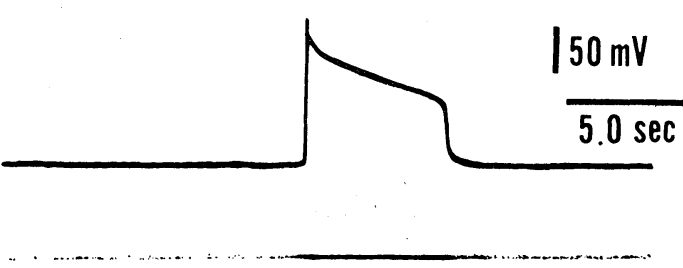

Fig. 4. Prolonged action potential (B) and the time course of sodium influx measured by the moving-autoradiographic method (C). An axon was perfused with $25 \mathrm{mM}$ CsF-glycerol solution internally and the extenal medium was $100 \mathrm{mM} \mathrm{CaCl}_{2}$ and $300 \mathrm{mM} \mathrm{NaCl}$. 
potential. The increase in the influx during the initiation of the prolonged action potential is continued during the plateau phase. Similar results were obtained with the influx of $\mathrm{Ca}^{45}$ with mixed solution of $100 \mathrm{mM} \mathrm{CaCl}_{2}$ and $300 \mathrm{mM} \mathrm{NaCl}$ and with $100 \mathrm{mM} \mathrm{CaCl}_{2}$ alone.

Discussion. The excitable membrane of the squid axon is thought to be composed of a complex macromolecular structure made up of proteins and phospholipids. According to the theory of excitation described in recent articles, ${ }^{1), 6)}$ the process of excitation is a manifestation of a transition of the membrane macromolecules from one stable conformational state (resting state) to a different conformational state (excited state). An abrupt change in the conformational state of the membrane macromolecules would be expected to result in discontinuous changes in membrane properties. The variation in membrane potential, the increased cation fluxes and the fall in membrane impedance associated with excitation may be regarded as a sign of a discontinuous change in membrane properties.

Since the plateau phase of the prolonged action potential is considered to represent an excited state of the membrane macromolecules, ${ }^{1,6), 8)}$ it is reasonable to suppose that membrane properties will continue in the same state throughout the plateau phase. Thus one might expect that the quantity of cation influx to be the same value during plateau phase. Our experimental findings described under results provide evidence for this interpretation.

Acknowledgment. We wish to express our gratitude to Professors A. Watanabe and Y. Katuki for their help throughout the experiment. We also wish to thank Dr. F. D. Walker for his valuable criticism and correcting the manuscript. This work was supported by a grant from the Ministry of Education (Japan).

\section{References}

1) Cohen, L. B., R. D. Keynes, and B. Hille: Nature, 218, 438 (1968).

2) Hodgkin, A. L., and B. Katz: J. Physioì., 108, 37 (1949).

3) Keynes, R. D., and P. R. Lewis: J. Physiol., 113, 73 (1951).

4) Tasaki, I., A. Watanabe, and T. Takenaka: Proc. Nat. Acad. Sc. USA, 48, 1177 (1962).

5) Tasaki, I., I. Singer, and T. Takenaka: J. Gen. Physiol., 48, 1095 (1965).

6) Tasaki, I., and I. Singer: An. New York Acad. Sci., 137, 792 (1966).

7) Tasaki, I.: Nerve Excitalion. Thomas Springfield, Illinois (1968).

8) Takenaka, T., and S. Yamagishi: J. Gen. Physiol., 53, 81 (1969). 